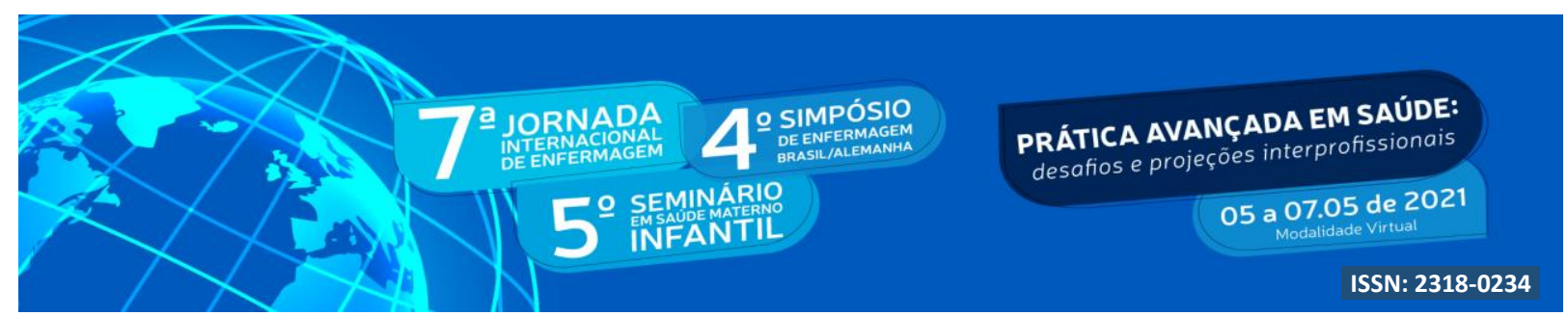

DOI: http://doi.org/10.48195/jie2021-069

\title{
A IMPORTÂNCIA DA COMUNICAÇÃO TERAPÊUTICA NO CUIDADO A PESSOA IDOSA COM CÂNCER APRESENTANDO MUCOSITE ORAL ${ }^{1}$
}

\author{
Betânia Huppes ${ }^{2}$; Julia Oliveira Silveira ${ }^{3}$; Morgana Aline da Silva ${ }^{4}$; Fabiana Porto da \\ Silva ${ }^{5}$; Naiana Oliveira dos Santos ${ }^{6}$; Maria Helena Gehlen ${ }^{7}$;
}

\begin{abstract}
RESUMO
A comunicação terapêutica é fundamental para identificar as necessidades do usuário, promover o conforto, contribuindo para melhores práticas de enfermagem. Vivênciamos a situação de uma idosa septuagenária com comprometimento na comunicação por conta da mucosite oral. De tal modo, foi promovido conforto por meio da comunicação terapêutica. Porém muitas vezes torna-se prejudicada junto aos pacientes idosos oncológicos que apresentam mucosite oral. Objetivo relatar a importância da comunicação terapêutica no cuidado realizado com uma pessoa idosa com câncer apresentando mucosite oral em um hospital público de baixa complexidade na cidade de Santa Maria - RS. Tratando-se de um relato de experiência, realizado na disciplina de Fundamentos Teóricos e Práticos de Enfermagem II, do curso de enfermagem de uma universidade privada do interior do Rio grande do Sul. Concluí-se que a comunicação terapêutica é fundamental para promover a atenção integral a saúde da pessoa idosa considerando a sua sinugularidade e bem estar.
\end{abstract}

Palavras-chave: Comunicação; Idoso; Oncologia; Terapêutica.

\begin{abstract}
Therapeutic communication is essential to identify the user's needs, promote comfort, contributing to better nursing practices. We experienced the situation of a septuagenarian elderly woman with impaired communication due to oral mucositis. In such a way, comfort was promoted through therapeutic communication. However, it often becomes impaired with elderly cancer patients who have oral mucositis. Objective to report the importance of therapeutic communication in the care provided to an elderly person with cancer presenting oral mucositis in a low complexity public hospital in the city of Santa Maria - RS. This is an experience report, carried out in the discipline of Theoretical and Practical Foundations of Nursing II, of the nursing course at a private university in the

\footnotetext{
1 Trabalho vivênciado na disciplina de Fundamentos Teóricos e Práticos da Enfermagem II do curso de Enfermagem - UFN.

${ }^{2}$ Acadêmica de enfermagem - UFN. betania.huppes@ufn.edur.br

${ }^{3}$ Acadêmica de enfermagem - UFN. julia.silveira@ufn.edu.br

${ }^{4}$ Acadêmica de enfermagem - UFN. morgana.a.dasilva@gmail.com

${ }^{5}$ Orientadora, Professora do Curso de Enfermagem - UFN. fabiana.silva@ufn.edu.br

${ }^{6}$ Orientadora, Professora do Curso de Enfermagem - UFN. naina_santos@ufn.edu.br
}

${ }^{7}$ Orientadora, Professora do Curso de Enfermagem - UFN. gehlenmh@gmail.com
\end{abstract}




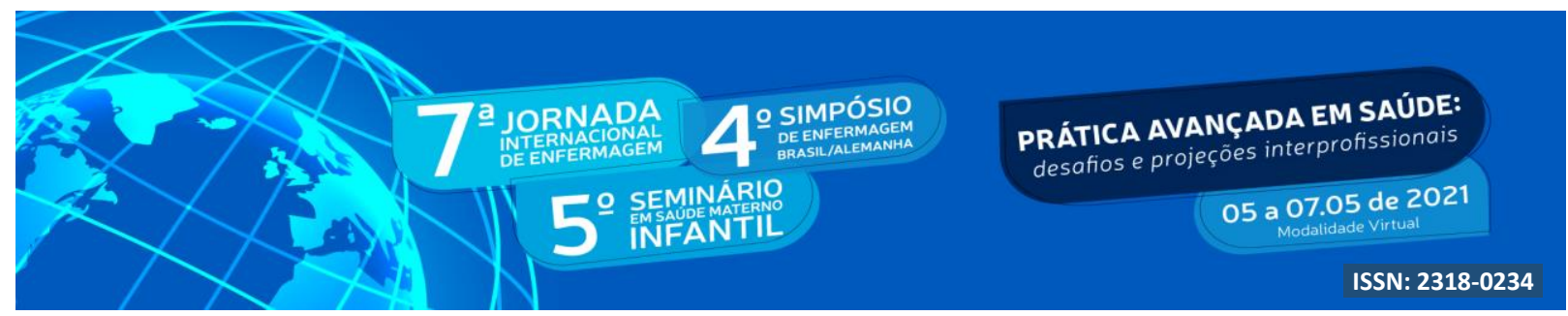

interior of Rio Grande do Sul. We conclude that therapeutic communication is fundamental to promote care integral to the health of the elderly considering their synugularity and well-being.

Key Words: Communication; Elderly; Oncology; Therapeutic.

\section{INTRODUÇÃO}

A comunicação é fundamental na interação humana, pois ela transmite pensamentos, valores e informações, no qual poderá ser expressa através da fala, gestos, expressões e entonações de palavras. Desta forma, está presente em todos os atendimentos de saúde, permitindo a criação do vínculo entre profissional da saúde e paciente, sendo fundamental para de informações importantes na anamnese, com conforto e bem-estar, principalmente diante de diagnósticos dolorosos e assustadores como o câncer (SILVA; BARROS, 2015).

Nesse sentido, torna-se fundamental o desenvolvimento da comunicação terapêutica principalmente para as pessoas idosas com mucosite oral em consequência de uma carcinogênese. Apesar do diagnóstico de câncer não ser uma sentença de morte, ele ainda é visto pela sociedade como uma doença incurável. Consequentemente, a notícia do diagnóstico da doença provoca um turbilhão de sentimentos, medos e conflitos internos. Assim, além da dor física, muitas vezes o indivíduo convive com a dor psíquica e espiritual, podendo desenvolver transtorno de ansiedade e/ou depressão (SANTOS, 2017).

Segundo o Instituto Nacional do Câncer (BRASIL, 2018) o envelhecimento natural traz mudanças nas células, o que torna a população idosa mais vulnerável a desenvolver um processo cancerígeno. Até mesmo pelo fato das células estarem expostas a mais tempo a diferentes fatores de risco, todavia, nesta fase sendo mais comum o desenvolvimento do câncer.

Felizmente já existem alternativas para o tratamento da doença, como a quimioterapia, radioterapia e/ou transplante de medula óssea, podendo combinar mais de uma destas modalidades. Porém, as alternativas terapêuticas poderão causar mucosite oral que é uma reação tóxica inflamatória que além de provocar desconforto e dor intensa, dificultará deglutição e comunicação (ARAÚJO et al., 2015). Assim, para atender as necessidades de 


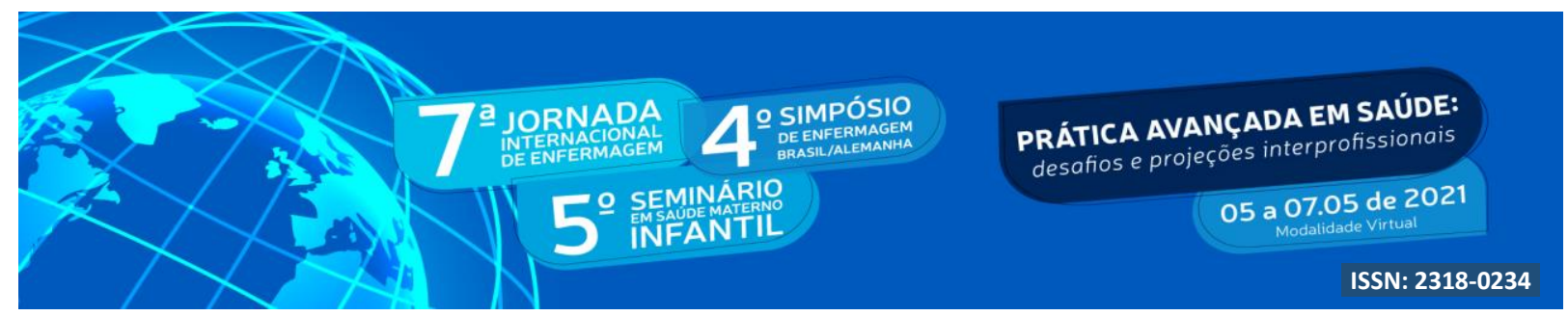

cuidado da pessoa idosa com mucosite oral, torna-se fundamental o desenvolvimento da comunicação terapêutica.

\section{OBJETIVO}

Relatar a importância da comunicação terapêutica no cuidado realizado com uma pessoa idosa com câncer apresentando mucosite oral em um hospital público de baixa complexidade na cidade de Santa Maria - RS

\section{METODOLOGIA}

Trata-se de um relato de experiência, realizado na disciplina de Fundamentos Teóricos e Práticos de Enfermagem II, do curso de enfermagem da Universidade Franciscana, no cenário hospitalar público de baixa complexidade localizado no interior do Rio Grande do Sul. No mês de outubro de 2020.

O relato de experiência possui uma enorme responsabilidade social, pois o artigo apresentará sua experiência em determinado local e data. Consequentemente, também poderá inspirar demais profissionais a partir das reflexões e intervenções positivas (TOSTA et al., 2016).

Desta forma ao se operacionalizar as aulas práticas da disciplina de fundamentos teóricos e práticos de enfermagem II, aliou-se os conhecimentos das disciplinas de Atenção Integral à Pessoa Idosa e Oncologia em Enfermagem o que permitiu nos efetivar a comunicação terapêutica para a pessoa idosa com mucosite oral ao se obter as melhores formas de estabelecer/criar intervenções de conforto, bem-estar, alívio da dor e do medo.

\section{RESULTADOS E DISCUSSÃO}

Com a integração das disciplinas de Fundamentos Teóricos e Práticos II, Atenção Integral à Saúde do Idoso e Oncologia em Enfermagem, foi possível promover o cuidado humanizado de enfermagem, promovendo o conforto, por meio da comunicação terapêutica.

A comunicação terapêutica foi essencial para a efetividade do cuidado humanizado. Com o vínculo estabelecido com a pessoa idosa com mucosite oral identificou-se as reais 


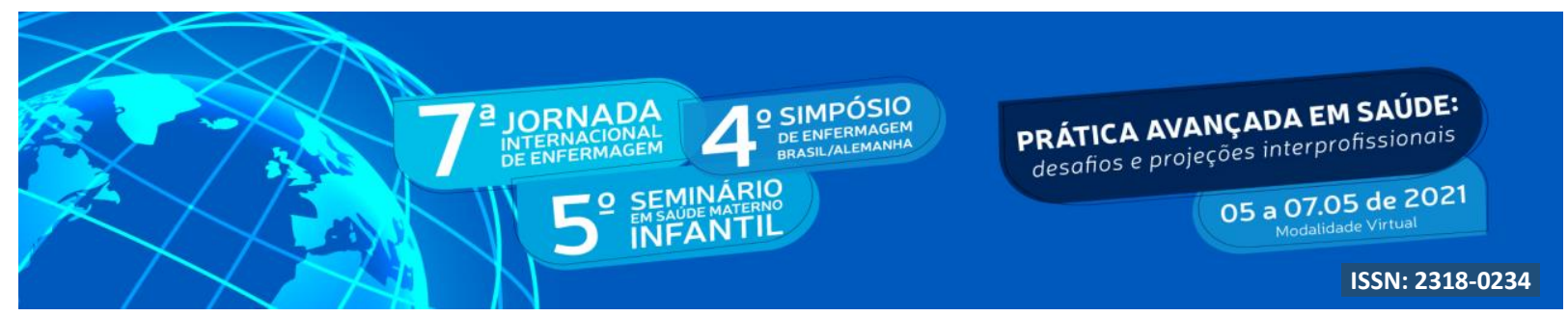

necessidades no período de hospitalização. Pois o vínculo permite que a pessoa idosa expresse seus desejos de conforto, já que considera-se não somente uma patologia, os usuários desejam uma simples conversa para distraí-los, um toque e por conta da rotina estressante de muitos profissionais, este cuidado por vezes é deixado de lado, valorizando somente as intervenções práticas e invasivas (TORRES et al., 2016).

Como é o caso da nossa paciente de 76 anos que estava internada e não conseguia se comunicar por conta da mucosite oral causada pelo tratamento oncológico. A acompanhante relata que a idosa sempre gostou de conversar, porém, nos últimos meses, é ignorada. Os profissionais pouco se comunicavam a mesma ao realizar os procedimentos, pois ela não conseguia verbalizar de forma entendível e rápida.

Ao realizarmos o cuidado de enfermagem, percebe-se que a pessoa idosa apresentava com a oxigenioterapia e a sonda nasogástrica. Então para minimizar o conforto prejudicado, desenvolveu-se a promoção da saúde por meio do diálogo esclarecedor quanto a necessidade dos dispositivos para o alivio da dor.

Na promoção dos cuidados da higiene facial, a comunicação terapêutica transcorreu quanto a temática de que estava um sábado muito bonito e ensolarado. Ao ver que a idosa se estimulava foi notável que havia entendimento. Foi questionado quanto o que mais gostava de fazer em casa, e surpreendentemente houve resposta verbal no que tange ao gostar de cozinhar e costurar, e com um sorriso demonstrou conforto e bem-estar.

Segundo o livro Diagnósticos de enfermagem da NANDA-I foi possível elencar no início da promoção dos cuidados de enfermagem os seguintes diagnósticos: Dor intensa e Conforto prejudicado por conta da mucosite oral que atrapalhava sua comunicação. Nesta perspectiva, implementou-se a da comunicação terapêutica integrada ao uso dos analgésicos. Esta efetivação, mantém a atenção integral a saúde da pessoa idosa de forma singular, centrado na pessoa, valorizando suas necessidades, lhe oferecendo as informações de forma adequada, objetiva e compreensível, sempre respeitando sua decisão ou da família, caso paciente não esteja consciente (ATTY; TOMAZELLI, 2018). 


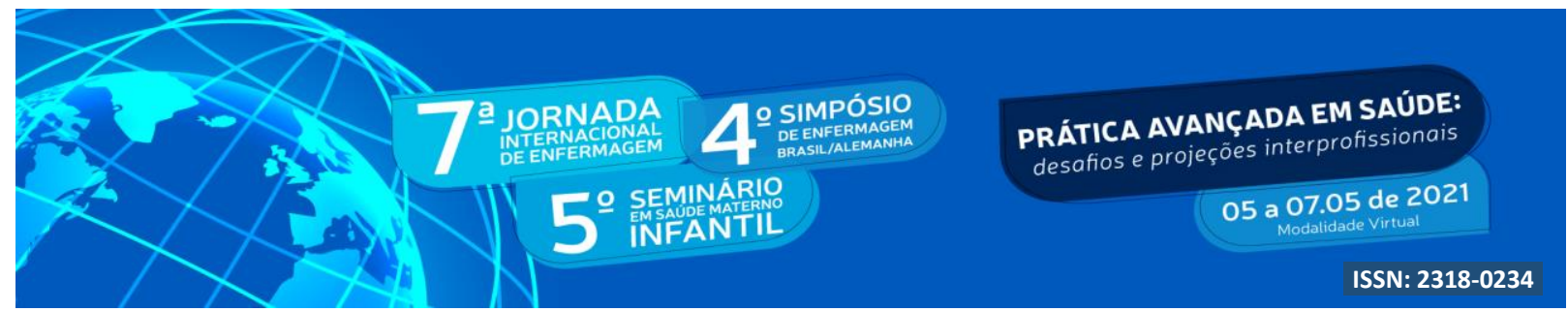

Vale ressaltar que por vezes a pessoa idosa não deseja conversar, por simplesmente não sentir-se bem ou a vontade. O silêncio também é uma forma de se comunicar e respeitar o tempo e espaço deste usuário. Nosso toque e olhar/expressões também poderão ser comunicações terapêuticas (JALLES; SANTOS; REINALDO, 2017). Cabe compreender e respeitar a singularidade para oferecer o melhor cuidado.

Apesar da comunicação terapêutica ser uma característica forte da equipe de enfermagem, um estudo realizado por ARAÚJO et al. (2015, p.272), evidenciou-se a carência de intervenções de enfermagem diante de pacientes oncológicos que apresentam mucosite oral, seja no serviço público como no serviço privado. Deste modo, reforça-se a importância da busca de fundamentos científicos que possam auxiliar e promover um atendimento adequado as pessoas iodas com mucosite, compreendendo as principais necessidades e fatores de risco. Consequentemente a comunicação terapêutica efetiva-se auxiliando na recuperação das patologias e fortalecendo o enfrentamento psicológico em relação ao câncer.

\section{CONCLUSÃO}

Conclui-se a partir deste relato de experiência, que a comunicação terapêutica é fundamental no tratamento da pessoa idosa com mucosite oral pois foi possível evidenciar que a comunicação, seja ela verbal ou não verbal, é fundamental para tranquilizar e consequentemente promover o conforto e auxiliar no controle da dor.

Por consequência, ao relatar a experiência da importância da comunicação terapêutica deseja-se incentivar a busca do conhecimento científico sobre a mucosite oral em pessoas idosas oncológicos, para proporcionar atendimentos dignos, mais acolhedores, integrais e humanizados.

\section{REFERÊNCIAS}

ARAUJO, S. N. M. et al. O paciente oncológico com mucosite oral: desafios para o cuidado de enfermagem.. Rev. Latino-Am. Enfermagem, Ribeirão Preto , v. 23, n. 2, p. 267-274, abr./2015. Disponível em: 


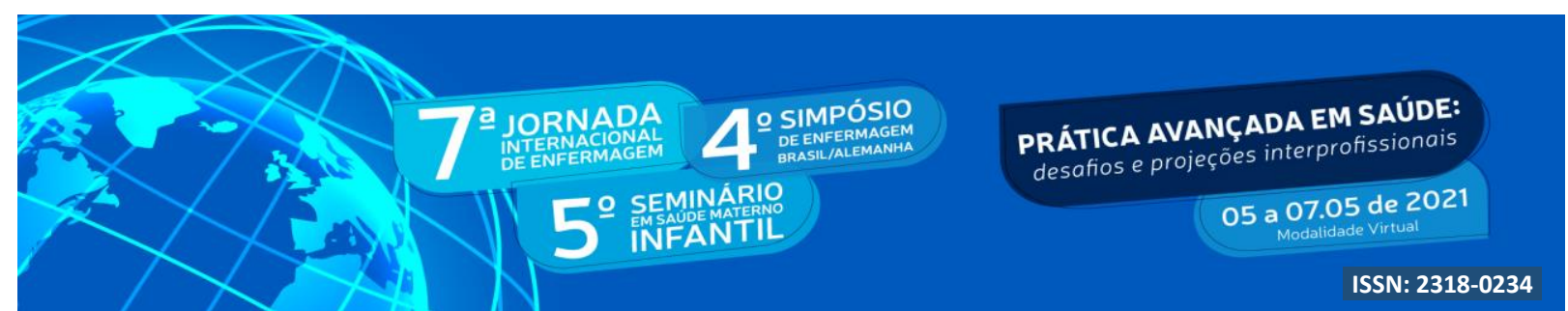

https://www.scielo.br/pdf/rlae/v23n2/pt_0104-1169-rlae-23-02-00267.pdf. Acesso em: 10 mar. 2021.

ATTY, A. T. D. M.; TOMAZELLI, J. G. Cuidados paliativos na atenção domiciliar para pacientes oncológicos no Brasil. Revista Saúde debate, Rio de Janeiro, v. 42, n. 116, p. 225 236, jan./2018. Disponível em: http://www.scielo.br/scielo.php?script=sci_arttext\&pid=S0103$11042018000100225 \& \operatorname{lng}=$ en\&nrm=iso. Acesso em: 10 mar. 2021.

Brasil. Ministério da Saúde. Instituto Nacional de Câncer. O que causa o câncer? Rio de Janeiro: INCA; 2018. Disponível em: https://www.inca.gov.br/causas-e-prevencao/o-quecausa-cancer (Acessado em 11 de março de 2021).

BROCA, P. V.; FERREIRA, M. D. A. Equipe de enfermagem e comunicação: contribuições para o cuidado de enfermagem. Rev. Brasileira de Enfermagem, Brasília, v. 65, n. 1, p. 97 103, fev./2012. Disponível em: http://www.scielo.br/scielo.php?script=sci_arttext\&pid=S003471672012000100014\&lng=en\&nrm=iso. Acesso em: 14 mar. 2021.

Diagnósticos de enfermagem da NANDA-I: definições e classificação 2018-2020. Traduzido por GARCEZ, R. M. et al. 11. ed. Porto Alegre: Artmed, 2018. p. 889-903.

JALLES, M. P.; SANTOS, V. S. J. D. REINALDO, A.M.D.S. Análise da produção científica sobre comunicação terapêutica no campo da saúde, saúde mental e álcool e outras drogas. Rev Med , São Paulo, v. 96, n. 4, p. 232-240, out./2017. Disponível em: https://www.revistas.usp.br/revistadc/article/download/122431/136719 . Acesso em: 13 mar. 2021.

SANTOS, M. A. D. Câncer e suicídio em idosos: determinantes psicossociais do risco, psicopatologia e oportunidades para prevenção. Ciênc. saúde coletiva, Rio de Janeiro, v. 22, n. $9, \quad$ p. 3061-3075, set./2017. Disponível em: 


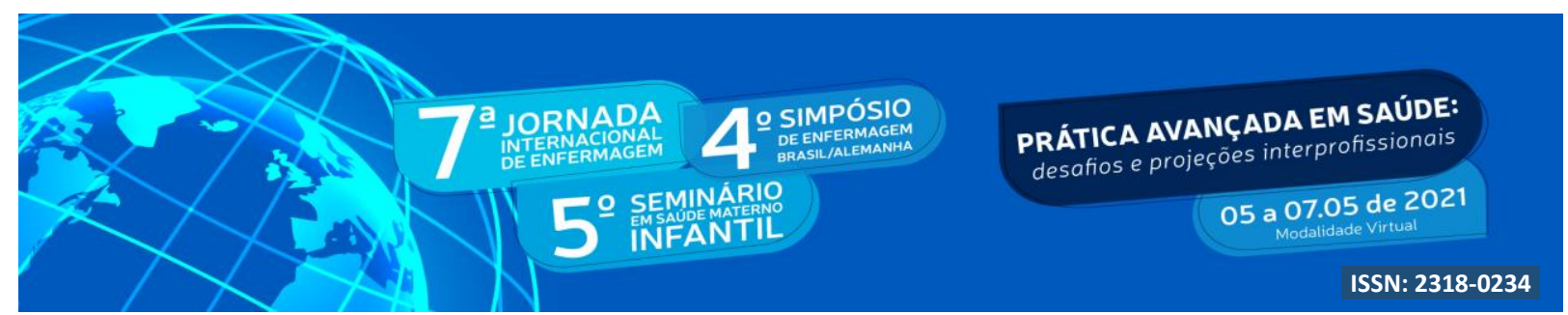

http://www.scielo.br/scielo.php?script=sci_arttext\&pid=S1413-

81232017002903061\&lng=en\&nrm=iso. Acesso em: 14 mar. 2021.

SILVA, R. C. D.; BARROS, C.V. D. L. Comunicação terapêutica relacionada ao cuidado humanizado e a segurança do paciente em unidade hospitalar. Saúde \& Ciência em Ação, São Paulo, v. 1, n. 1, p. 13-25, jul./2015. Disponível em: https://revistas.unifan.edu.br/index.php/RevistaICS/article/view/110. Acesso em: 9 mar. 2021.

TORRES, G. M. C. et al. Comunicação terapêutica na interação profissional de saúde e hipertenso na estratégia saúde da família. Rev Gaúcha Enferm., Porto Alegre, v. 38, n. 4, p. 1-8, ago./2017. Disponível em: https://www.scielo.br/pdf/rgenf/v38n4/1983-1447-rgenf-3804-e2016-0066.pdf. Acesso em: 14 mar. 2021.

TOSTA, L. R. D. O.; SILVA, T. B. F; SCORSOLINI-COMIN, F. O Relato de Experiência Profissional e sua Veiculação na Ciência Psicológica. Revista Clínica \& Cultura, Sergipe, v. 2, n. $1, \quad$ p. 62-73, jul./2016. Disponível em: https://seer.ufs.br/index.php/clinicaecultura/article/download/6016/5994. Acesso em: 10 mar. 2021. 